\title{
Abstract: Chemisorption on a model bcc metal ${ }^{* \dagger}$
}

\author{
W. Ho, S. L. Cunningham $\ddagger$, and W. H. Weinberg \\ Division of Chemistry and Chemical Engineering, California Institute of Technology, Pasadena, California 91125
}

(Received 4 September 1975; in final form 2 October 1975)

PACS numbers: 82.65.M, 73.20.

The system considered here is that of a single atom with one energy level chemisorbed on the (001) surface of a model bcc metal. We present the change in the density of electronic states $\Delta n(E)$ due to chemisorption for two cases: one when the adatom is bound to a single substrate atom in the "on-site" configuration and the other when it is bound to four substrate atoms in the "centered fourfold site." In principle, this change in the density of states $\Delta n$ can be related to the results of photoemission measurements. ${ }^{1}$

There are four reasons for this study: (1) By comparing with previous results ${ }^{2}$ of chemisorption on the (001) surface of a model simple-cubic metal, we can determine the sensitivity of $\Delta n(E)$ to the substrate crystal structure. (2) We can investigate the sensitivity of $\Delta n(E)$ to the binding site configuration. (3) The same model of the substrate can be easily extended to that of a two-band crystal with the $\mathrm{CsCl}$ structure. ${ }^{3}$ Therefore the results of this work will serve as a basis for comparison with the results of a chemisorption study on a model insulator surface. ${ }^{4}$ (4) Finally, a simple modification of the present theory can be used to study the adsorption of a monolayer of atoms on the same surface. $^{4}$ A comparison of $\Delta n(E)$ calculated for a single chemisorbed atom with $\Delta n(E)$ for a monolayer will be important for understanding experimental photoemission results.

To model the bcc metal substrate, we use the linear combination of atomic orbitals (LCAO) method and the tight binding approximation (TBA) to generate the electronic energy levels and wave functions. Each atom is assumed to have one orbital at energy $E_{1}$, and only nearest-neighbor overlap integrals $\gamma$ are considered. A complete description of the Green's functions appropriate for the (001) surface of this model metal has been presented elsewhere. ${ }^{3}$ For the adatom, we assume a single energy level of $E_{a}$ coupled to the surface with an interaction strength $\sigma$. The Green's function for this single level system is well known. ${ }^{2}$

To obtain the change in the electronic density of states due to chemisorption, we follow the procedures used before ${ }^{2,5}$ of determining the phase shift due to the perturbation potential associated with the formation of the chemisorption bond. This phase shift is the negative of the argument of the complex determinant of the matrix I-VG, where $I$ is the unit matrix, $V$ is the perturbation matrix, and $G$ is a matrix containing the Green's functions for the adsorbed atom and the clean crystal surface. The change in the density of states $\Delta n(E)$ is related simply to the derivative with respect to energy of this phase shift.

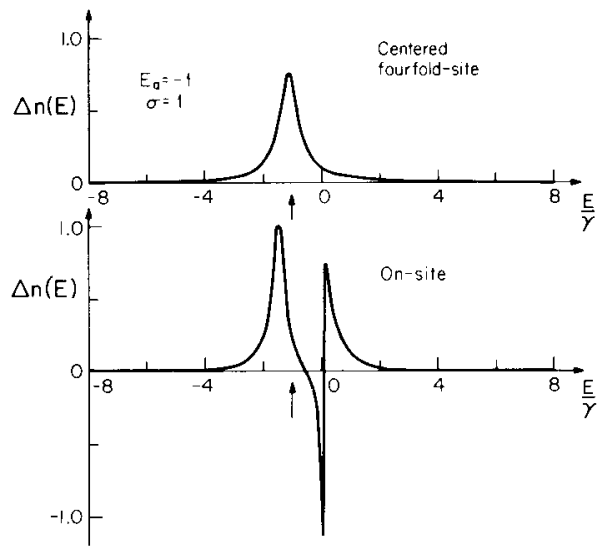

FIG. 1. The change in the density of electronic states as a function of energy due to the chemisorption of a single atom of energy $E_{a}=-1$ on the (001) surface of a model bcc metal in two different binding sites. All energies are in units of $\gamma$, the metal nearestneighbor hopping integral. The case shown represents weak binding.

In Fig. 1 we present a sample of the results. We plot the change in the density of states $\Delta n$ as a function of energy $E$, where all energies are expressed in units of $\gamma$. In these units the band width is 16 . We have arbitrarily chosen the zero of energy to be at the middle of the band. In Fig. 1 we have set the adatom energy level $E_{a}=-1$ as shown by the arrow. The binding strength is chosen to be $\sigma=1$. (Note that $\sigma$ is the overlap integral of the adatom orbital with the total surface wave function to which it binds. For on-site adsorption, this is the same as the nearestneighbor hopping integral, but for the centered fourfold-site adsorption it is different. See Ref. 5.)

For the on-site adsorption, $\Delta n$ shows a bonding and antibonding resonance with a depletion of states near the middle of the band. This behavior occurs for all values of $\sigma$ (even very small) and all values of $E_{a}$. As $\sigma$ increases, the resonances move apart in energy and eventually become splitoff states below and above the band. This unexpected behavior for small $\sigma$ is due to the fact that the surface Green's function appropriate for on-site adsorption is singular at $E=0$. (This implies that there is a singularity in the density of states of the unperturbed surface.)

For the centered-four-fold adsorption, the behavior is very similar to the results presented previously for the simple-cubic substrate. ${ }^{2}$ For small values of $\sigma(\sigma<2)$, the adatom level is a single broadened resonance. For stronger binding $(\sigma>2)$, the peak splits into a bonding and antibonding resonance and these move apart in 
energy as $\sigma$ increases until one or both of the resonances become splitoff states. The behavior of this adsorption site result differs from the on-site results for small $\sigma$ because the Green's function appropriate for the four-fold-site adsorption does not contain any singularities.

In conclusion, we have found that if the Green's function describing the substrate binding site contains a singularity, then the change in the density of states upon chemisorption shows a depletion of states at the energy of that singularity and this is independent of the strength of the coupling. For weak-binding chemisorption, this causes the change in the density of states to differ in a qualitative way depending on the substrate crystal structure (our on-site results differ from those for a simple cubic crystal substrate) or on the binding site geometry (our results differ for the two types of binding sites). Except for this one characteristic, however, the other features of the change in the density of states are not sensitive to either the substrate crystai structure or the binding site geometry, particularly for the case of strong chemisorption $(\sigma>5)$.

*Complete paper to appear in Surf. Sci.

$\dagger$ Work supported by NSF Grant GK-43433.

†IBM postdoctoral research fellow.

${ }^{1}$ See, for example, W.F. Egelhoff and D.L. Perry, Phys. Rev. Lett. 34, 93 (1975); D.E. Eastman and M.I. Nathan, Phys. Today 28, 44 (1975).

${ }^{2}$ T.L. Einstein, Surf. Sci. 45, 713 (1974); J.W. Davenport, T.L. Einstein, and J.R. Schrieffer, Jpn. J. Appl. Phys. Suppl. 2, Pt. 2, 691 (1974).

${ }^{3}$ W. Ho, S.L. Cunningham, W.H. Weinberg, and L. Dobrzynski, Phys. Rev. B 12, 3027 (1975).

${ }^{4}$ W. Ho, S.L. Cunningham, and W.H. Weinberg (unpublished).

${ }^{5}$ G. Allan, Ann. Phys. Paris 5, 12 (1970).

\title{
Abstract: Hydrogen and transition metal adatom chemisorption on transition metals as a function of $d$ bandfilling: Tenfold degenerate Hubbard and Anderson models
}

\author{
Edward Siegel \\ 24 Fifth Avenue, New York, New York 10011 \\ (Received 16 September 1975)
}

PACS numbers: 82.65.M, 73.20.

The Newns theory ${ }^{1}$ of the chemisorption of both hydrogen and transition metal adatoms via the Hubbard and Anderson models is reformulated in the true $d$-electron, tenfold spin-orbitally-degenerate Hubbard and Anderson models of Siegel and Kemeny. The theory is generally valid for chemisorption of hydrogen or any transition metal adatom on any transition metal surface, but we compare it for the Newns case of $5 d$ adatom chemisorption on tungsten. The agreement is seen to be better. We also compare this treatment with that of chemisorption of Schrieffer and Gomer ${ }^{2}$ and conclude that this non-screened, intra-atomic Hubbard or Anderson model treatment is better than their interatomic Hubbard or Anderson model treatment because intra-atomic coulomb and exchange interactions should dominate even without electron screening to decrease any interatomic electron interaction. The explicit inclusion of Hunds rule in the Siegel-Kemeny theory also has profound consequences for transition metal surfaces. Lastly, the superiority of the perturbation theory treatment of the problem over the Hartree-Fock approximation or vice-versa is explored. It is shown that this is so for transition metal adatom chemisorption but the reverse is true for hydrogen chemisorption.

${ }^{1}$ D. M. Newns, Phys. Rev. Lett. 25, 22 (1970).

${ }^{2}$ J. R. Schrieffer and R. Gomer, Surf. Sci. 25, 315 (1971). 